\title{
Plasma depletion layer: the role of the slow mode waves
}

\author{
Y. L. Wang ${ }^{1}$, J. Raeder ${ }^{2}$, and C. T. Russell ${ }^{3}$ \\ ${ }^{1}$ Los Alamos National Laboratory, Los Alamos, USA \\ ${ }^{2}$ University of New Hampshire, Durham, USA \\ ${ }^{3}$ University of California, Los Angeles, USA
}

Received: 26 November 2003 - Revised: 25 September 2004 - Accepted: 3 October 2004 - Published: 22 December 2004

\begin{abstract}
The plasma depletion layer (PDL) is a layer on the sunward side of the magnetopause with lower plasma density and higher magnetic field compared to their corresponding values in the upstream magnetosheath. The depletion layer usually occurs during northward interplanetary magnetic field (IMF) conditions with low magnetic shear across the magnetopause. We have previously validated the Raeder global model by comparing the computed formation of a magnetosheath density depletion with in-situ observations. We also have performed a detailed force analysis and found the varying roles that different MHD forces play along the path of a plasma parcel flowing around the magnetopause. That study resulted in a new description of the behavior of magnetosheath magnetic flux tubes which better explains the plasma depletion along a flux tube. The slow mode waves have been observed in the magnetosheath and have been used to explain the formation of the PDL in some of the important PDL models. In this study, we extend our former work by investigating the possible role of the slow mode waves for the formation of the PDL, using global MHD model simulations. We propose a new technique to test where a possible slow mode front may occur in the magnetosheath by comparing the slow mode group velocity with the local flow velocity. We find that the slow mode fronts can exist in certain regions in the magnetosheath under certain solar wind conditions. The existence and location of such fronts clearly depend on the IMF. We do not see from our global simulation results either the sharpening of the slow mode front into a slow mode shock or noticeable changes of the flow and field in the magnetosheath across the slow mode front, which implies that the slow mode front is not likely responsible for the formation of the PDL, at least for the stable solar wind conditions used in these simulations. Also, we do not see the two-layered slow mode structures shown in some observations and proposed in certain PDL models. Instead, we see only a one-layered spatial PDL structure under the stable solar wind conditions used in this study.
\end{abstract}

Correspondence to: Y. L. Wang

(ywang@lanl.gov)

\section{Introduction}

The coupling between the solar wind and the Earth's magnetosphere is through the magnetosheath and the magnetopause. It is thus important to understand the dynamics in this region before we can better understand the Sun-Earth coupling and the global dynamics of the Earth's magnetosphere system. The plasma depletion layer (PDL) is a layer on the sunward side of the magnetopause. It differentiates itself from neighboring magnetosheath regions by increased magnetic field and decreased plasma density. The PDL usually occurs when the IMF is northward with low magnetic shear across the magnetopause, in which case the magnetic reconnection is weak, so that magnetic field lines pile up on the sunward side of the magnetopause. However, the PDL can also occur during southward IMF when the solar wind dynamic pressure is high, in which case the magnetic reconnection cannot compete with the piling up of magnetic field lines, thus forming the PDL structure (Anderson et al., 1997). The observation of the plasma depletion layer started as early as 1968 (Cummings and Coleman, 1968). It has been carried on until recently by many authors (e.g. Crooker et al., 1979; Fuselier et al., 1991; Song et al., 1993; Paschmann et al., 1993; Anderson and Fuselier, 1993; Anderson et al., 1994; Phan et al., 1994, 1997).

The first comprehensive theoretical plasma depletion layer study was conducted by Zwan and Wolf (1976), who investigated the evolution of a single thin flux tube moving from the solar wind into the magnetosheath. They proposed two processes for depleting the flux tube: first, by deflecting plasma around the magnetosphere, the bow shock pushes plasma out along the field lines away from the nose of the magnetosphere; second, the compressional stress exerted on magnetosheath flux tubes near the nose tends to squeeze plasma out along the field lines, further depleting them. In their study, they pointed out that their solutions correspond to the slow mode waves expanding along the flux tube. However, as pointed out by Southwood and Kivelson (1992), the function of the wave in moving plasma is contrary to the slow mode wave properties, i.e. moving plasma into the magnetopause nose instead of moving it out. 


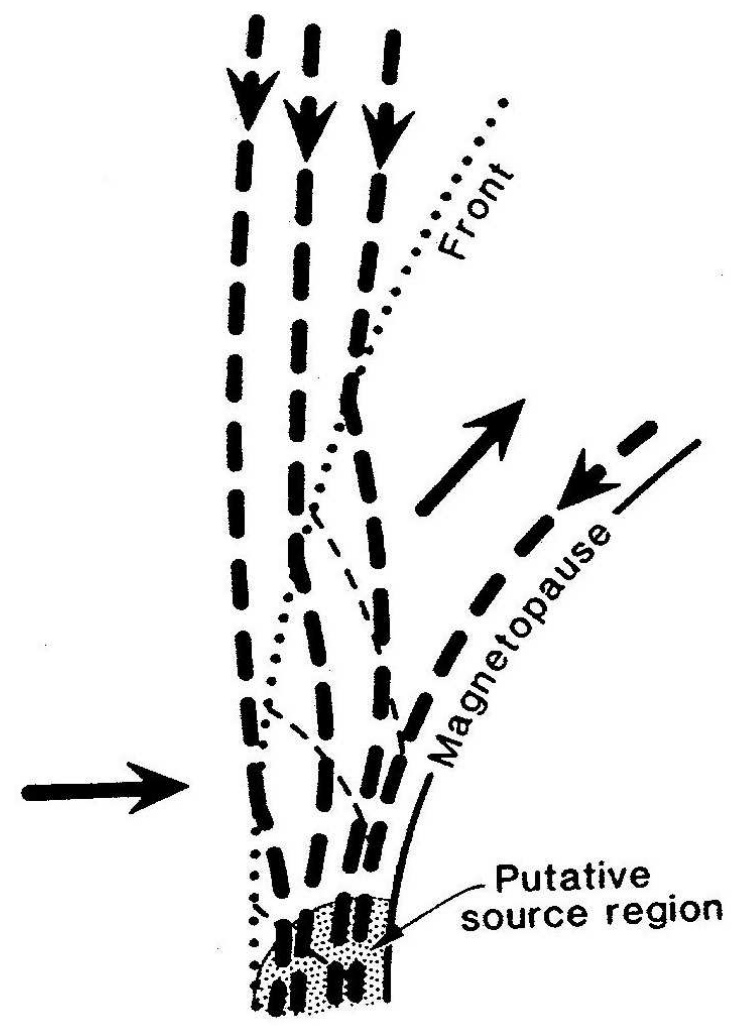

Fig. 1. Taken from Southwood and Kivelson (1992). A sketch shows the flow structure upstream of the subsolar magnetopause. There is a field compression region with roughly the properties of Zwan and Wolf (1976)'s flux tube immediately adjacent to the magnetopause. However, the field is not aligned with the outer boundary of the region. Field lines threading the compression regions bend toward the Sun and enter a field rarefaction region which is immediately behind a slow MHD wave front. Outside the front the field threads the incoming magnetosheath flow.

Using ISEE magnetosheath observations, Song et al. $(1990,1992)$ showed two-layered slow mode structures on the magnetopause, with decreased magnetic field magnitude and enhanced plasma density in the outer layer, and enhanced magnetic field magnitude and decreased plasma density in the inner layer. Based on the observations above, Southwood and Kivelson (1992) considered the formation of the plasma depletion layer from the point of view of MHD waves, as shown in Fig. 1. They pointed out that in general an obstacle in a plasma flow should excite all three MHD modes standing upstream from the obstacle: fast mode, slow mode, and intermediate mode waves. The fast mode waves form the bow shock. The other two modes should form fronts well inside the magnetosheath at the locations where the wave velocity matches the component of the magnetosheath velocity normal to the front. If the flow is to be diverted away from the stagnation streamline, the pressure must rise across the slow mode front. The field must drop if the pressure rises, or else the fast mode waves can arise and propagate away from the slow mode front. The density enhancement and field decrease after the slow mode front lead to the bending of the

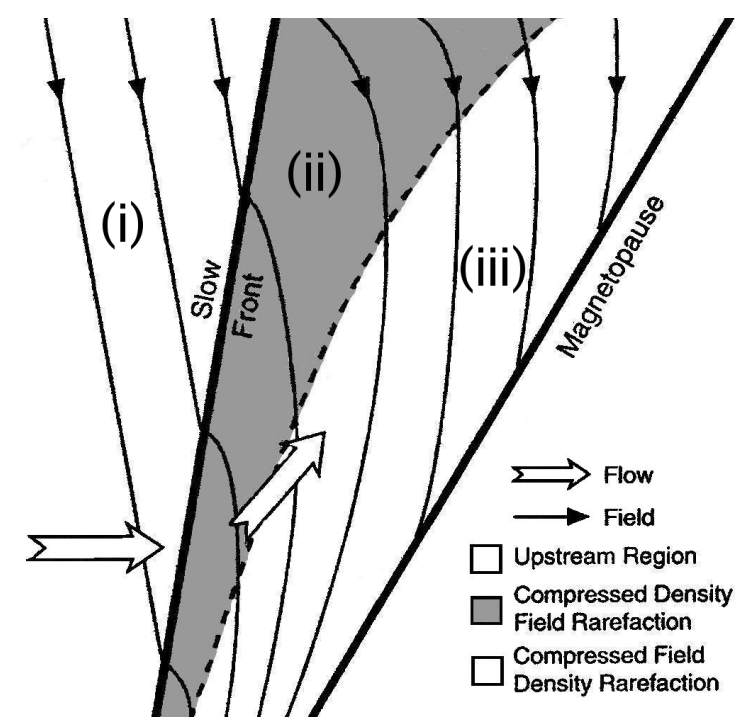

Fig. 2. A sketch of the basic structures of the subsolar magnetosheath flow taken from Southwood and Kivelson (1995). In the figure, there are structures with large physical parameter variations occurring at the bow shock, the magnetopause, the plasma depletion layer, and the slow wave front. They are all constrained to limited regions. For the other parts that occupy most of the volume of the magnetosheath, smooth flow and field dominate. Region (iii) is the conventional plasma depletion layer.

field toward the magnetopause nose after the front, which is farther away from the magnetopause boundary condition with tangential magnetic field on the magnetopause. Southwood and Kivelson (1992) proposed solving Bernoulli's flow equations to make the field and flow fit the specific magnetopause boundary conditions. They believed that this would lead to the conventional plasma depletion layer on the magnetopause. In this model, the inner boundary of the PDL is the magnetopause. However, no definite boundary is given between the PDL and the region with enhanced plasma density.

Later, Southwood and Kivelson (1995) tried to reconcile the discrepancies between the earlier Zwan and Wolf (1976) model and the Southwood and Kivelson (1992) model with a new scenario. They believed that the detached regions of enhanced density and the depletion layer adjacent to the magnetopause are manifestations of a single phenomenon. In their new scenario, as shown in Fig. 2, three regions were identified in the magnetosheath: (i) an upstream region where the flow is insufficiently deflected to move around the boundary; (ii) a frontal region where deflection of the flow is imposed; (iii) a region of depletion against the magnetopause. In these three regions, normal magnetosheath density and magnetic field, compressed density and rarefied magnetic field, and compressed magnetic field and rarefied density (the PDL) exist, respectively. The field lines are not aligned with the outer boundary of the plasma depletion region. The plasma compression results in a net force exerted on the plasma at the slow mode front which causes the deflection of the flow 
away from the nose of the magnetosphere. On those parts of the field line which crossed the front, there is motion away from the nose. This flow, induced by the slow front, gives rise to a density depletion near the nose. Thus, this new picture introduces into the Zwan and Wolf model a mechanism for squeezing the plasma along the field and compressing the magnetic flux to enhance the magnetic field. Just like a standing fast mode wave which has a compression (the bow shock) and an expansion fan along the flanks where the density decreases, the slow mode wave can also be associated with a compressional front and an expansion fan. Since the slow mode wave is slower than the fast mode wave, the slow mode structure must appear much closer to the magnetopause. Also, because the slow mode wave is guided by the magnetic field and cannot propagate in any direction like the fast mode wave, it must have a restricted region of occurrence and thus be more difficult to observe. Although the Southwood and Kivelson (1995) model gives a reasonable description of the possible structure and possible underlying physics for the formation of the PDL, their explanation depends on the solar wind and IMF conditions, as well as the propagation of the slow mode waves in the complex flow and field geometry in the magnetosheath. It is not clear whether the slow mode front can develop into a slow mode shock or can significantly change the property of plasma and field in the magnetosheath across this front, or if it is just too weak to play an important role. Meanwhile, other possible processes, for example, fast mode expansion, would compete with a slow mode shock. The final pattern in the magnetosheath will depend on the relative significance of different processes.

To avoid the difficulties of observational and theoretical studies in investigating the plasma depletion layer, numerical simulations have been applied to address the PDL problems (e.g. Wu, 1992; Lyon, 1994; Denton and Lyon, 2000). Recently, Siscoe et al. (2002) summarized four important MHD effects in the magnetopause boundary layer and the magnetosheath that cannot be produced by gasdynamic models, one of which is the PDL. They found a clear dependence of the PDL thickness on the IMF clock angle based on their ISM global model results. Wang et al. (2003) compared the Raeder global MHD model results, using IMP 8 and ACE solar wind plasma and IMF observations as a driver, with Wind in-situ observations for two PDL events on 12 January 1996 and 1 January 1999. They found good visual consistency between PDL observations and their model results. Meanwhile, their average model departures, which are defined as the average separations between model results and observations, are generally smaller than the standard deviations of observations. They concluded that an MHD description is sufficient to produce the plasma depletion layer. Furthermore, they showed that the PDL is stable for stable solar wind conditions and that small magnetosheath variations observed by Wind were temporal, rather than spatial, variations during these two events. Wang et al. (2004) further studied the detailed forces responsible for the formation of the PDL in the magnetosheath, using results from the Raeder global model.
They found that MHD forces play different roles at different locations along the path of a plasma parcel flowing around the magnetopause to form the PDL. Further, they gave a new and more detailed description of the plasma depletion in a flux tube inside the magnetosheath which is different from the two-step flux tube plasma density depletion proposed by Zwan and Wolf (1976).

In this paper we go one step further and use the Raeder global MHD model as a tool to investigate whether the slow mode front can exist in the magnetosheath, and, if so, what role it plays in the formation of the PDL. First, we give a brief introduction to the basics of the slow mode waves and propose a new numerical method to test the existence of the slow mode front in the magnetosheath. We then briefly discuss the Raeder global model, as well as the major model inputs used in this study. After that, we show some model results and discuss the implications of those results for the questions that we want to answer in this study. Finally, we summarise our results and clarify the role of the slow mode waves for the formation of the PDL.

\section{Theory}

As discussed above, the MHD slow mode waves have been invoked for the formation of the PDL, both in observations by Song et al. $(1990,1992)$ and in theoretical studies by Zwan and Wolf (1976) and Southwood and Kivelson (1992, 1995). In this section, we first discuss some of the important features of the slow mode waves before we use those features to develop a slow mode front detection technique and address the role of the slow mode waves in PDL formation.

The dispersion relation of the slow mode wave for a uniform background is:

$$
\frac{\omega^{2}}{k^{2}}=\frac{1}{2}\left\{\mathrm{C}_{\mathrm{s}}^{2}+\mathrm{V}_{\mathrm{A}}^{2}-\sqrt{\left(\mathrm{C}_{\mathrm{S}}^{2}+\mathrm{V}_{\mathrm{A}}^{2}\right)^{2}-4 \mathrm{C}_{\mathrm{S}}^{2} \mathrm{~V}_{\mathrm{A}}^{2} \cos ^{2} \theta}\right\},
$$

where $\theta$ is the angle between the slow mode wave normal, $\boldsymbol{k}$, and the background magnetic field, $\boldsymbol{B}, \mathrm{C}_{\mathrm{s}}$ is the sound speed, and $\mathrm{V}_{\mathrm{A}}$ is the Alfvén speed. The group velocity of the slow mode wave can be obtained by calculating $\partial \omega / \partial \boldsymbol{k}$, whose parallel and perpendicular components to the background magnetic field are:

$$
\begin{aligned}
& V_{g \|}=\frac{\partial \omega}{\partial k_{\|}}= \\
& \frac{\left(\mathrm{C}_{\mathrm{s}}^{2}+\mathrm{V}_{\mathrm{A}}^{2}\right) \cos \theta-\frac{\left(\mathrm{C}_{\mathrm{S}}^{2}+\mathrm{V}_{\mathrm{A}}^{2}\right)^{2} \cos \theta-2 \mathrm{C}_{\mathrm{S}}^{2} \mathrm{~V}_{\mathrm{A}}^{2}\left(2 \cos ^{3} \theta+\cos \theta \sin ^{2} \theta\right)}{\sqrt{\left(\mathrm{C}_{\mathrm{S}}^{2}+\mathrm{V}_{\mathrm{A}}^{2}\right)^{2}-4 \mathrm{C}_{\mathrm{s}}^{2} \mathrm{~V}_{\mathrm{A}}^{2} \cos ^{2} \theta}}}{\sqrt{2} \sqrt{\left(\mathrm{C}_{\mathrm{s}}^{2}+\mathrm{V}_{\mathrm{A}}^{2}\right)-\sqrt{\left(\mathrm{C}_{\mathrm{S}}^{2}+\mathrm{V}_{\mathrm{A}}^{2}\right)^{2}-4 \mathrm{C}_{\mathrm{S}}^{2} \mathrm{~V}_{\mathrm{A}}^{2} \cos ^{2} \theta}}}, \\
& V_{g \perp}=\frac{\partial \omega}{\partial k_{\perp}}= \\
& \frac{\left(\mathrm{C}_{\mathrm{S}}^{2}+\mathrm{V}_{\mathrm{A}}^{2}\right) \sin \theta-\frac{\left(\mathrm{C}_{\mathrm{S}}^{2}+\mathrm{V}_{\mathrm{A}}^{2}\right)^{2} \sin \theta-2 \mathrm{C}_{\mathrm{S}}^{2} \mathrm{~V}_{\mathrm{A}}^{2} \sin \theta \cos ^{2} \theta}{\sqrt{\left(\mathrm{C}_{\mathrm{s}}^{2}+\mathrm{V}_{\mathrm{A}}^{2}\right)^{2}-4 \mathrm{C}_{\mathrm{S}}^{2} \mathrm{~V}_{\mathrm{A}}^{2} \cos ^{2} \theta}}}{\sqrt{2} \sqrt{\left(\mathrm{C}_{\mathrm{S}}^{2}+\mathrm{V}_{\mathrm{A}}^{2}\right)-\sqrt{\left(\mathrm{C}_{\mathrm{S}}^{2}+\mathrm{V}_{\mathrm{A}}^{2}\right)^{2}-4 \mathrm{C}_{\mathrm{S}}^{2} \mathrm{~V}_{\mathrm{A}}^{2} \cos ^{2} \theta}}},
\end{aligned}
$$



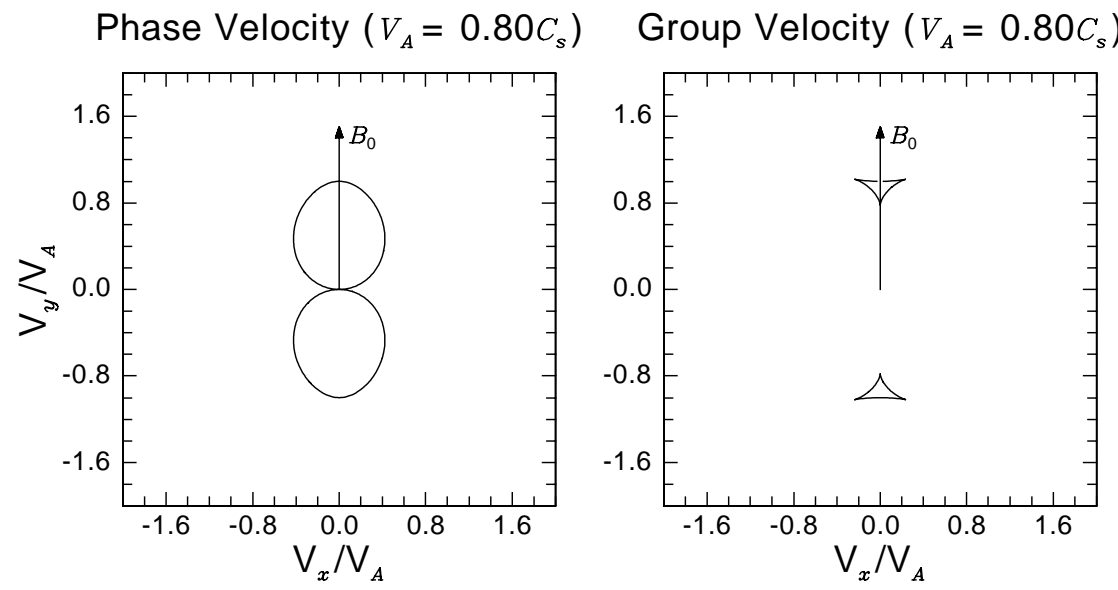

Fig. 3. The slow mode wave phase and group velocities relative to the background magnetic field for $V_{A}=0.8 C_{S}$. Here $V_{A}$ is the Alfvén speed, $\mathrm{C}_{\mathrm{S}}$ is the sound speed, and $\boldsymbol{B}_{0}$ is the background magnetic field. Although the phase velocity can be in any direction except perpendicular to the background magnetic field, the group velocity can only be within a small angle away from the background magnetic field.

where $\theta$ is the same as that used in Eq. (1), and it is not the angle between the slow mode wave group velocity and the background magnetic field. The angle between the slow mode wave group velocity and the background magnetic field can be calculated by

$\theta_{g}=\arctan \left(\frac{V_{g \perp}}{V_{g \|}}\right)$

When $\cos \theta$ approaches 0, Eqs. (2) and (3) become

$$
\begin{aligned}
V_{g \|} & =\frac{\mathrm{C}_{\mathrm{s}} \mathrm{V}_{\mathrm{A}}}{\sqrt{\mathrm{C}_{\mathrm{s}}^{2}+\mathrm{V}_{\mathrm{A}}^{2}}} \frac{\cos \theta}{|\cos \theta|}, \\
V_{g \perp} & =0 .
\end{aligned}
$$

From Eq. (5) we see that there are two singular points for $V_{g \|}: \theta=90^{\circ}$ and $\theta=270^{\circ}$.

- When $\theta$ approaches $90^{\circ}$ from a smaller angle,

$$
V_{g \|}=\frac{\mathrm{C}_{\mathrm{s}} \mathrm{V}_{\mathrm{A}}}{\sqrt{\mathrm{C}_{\mathrm{S}}^{2}+\mathrm{V}_{\mathrm{A}}^{2}}} \text {. }
$$

- When $\theta$ approaches $90^{\circ}$ from a larger angle,

$$
V_{g \|}=-\frac{\mathrm{C}_{\mathrm{S}} \mathrm{V}_{\mathrm{A}}}{\sqrt{\mathrm{C}_{\mathrm{S}}^{2}+\mathrm{V}_{\mathrm{A}}^{2}}} \text {. }
$$

- When $\theta$ approaches $270^{\circ}$ from a smaller angle,

$$
V_{g \|}=-\frac{\mathrm{C}_{\mathrm{s}} \mathrm{V}_{\mathrm{A}}}{\sqrt{\mathrm{C}_{\mathrm{s}}^{2}+\mathrm{V}_{\mathrm{A}}^{2}}} \text {. }
$$

- When $\theta$ approaches $270^{\circ}$ from a larger angle,

$$
V_{g \|}=\frac{\mathrm{C}_{\mathrm{s}} \mathrm{V}_{\mathrm{A}}}{\sqrt{\mathrm{C}_{\mathrm{S}}^{2}+\mathrm{V}_{\mathrm{A}}^{2}}} \text {. }
$$

The slow mode phase and group velocities from Eqs. (1-3) for $\mathrm{V}_{\mathrm{A}}=0.8 \mathrm{C}_{\mathrm{s}}$ are shown in Fig. 3 . The phase velocity stands for the wave front velocity of a planar wave, and it can be in any direction except perpendicular to the background magnetic field. The group velocity stands for the energy propagation velocity and it can only be within a small angle away from the background magnetic field. For planar waves, we can simply use Eq. (1) to calculate the velocity of the wave front. However, for non-planar waves, the group velocity should be applied instead for each slow mode wave point source. The envelope of the wave fronts from a group of point sources should be the same as what we can obtain from Eq. (1) when the point sources are planar. However, for nonplanar wave sources, like the slow mode wave sources in the magnetosheath, the point source solution for every point should be used to account for the complex source geometry. We will use this fact later to introduce a new technique to detect the slow mode front in the magnetosheath.

One way to see how the slow mode wave, with a point source, propagates in a plasma environment is through a simple numerical simulation. Such a test, if properly set, can also serve to validate the simulation code that we will later use to address the role of the slow mode waves in the magnetosheath. Such a simulation is done in a box with uniform background plasma and magnetic field. For the purpose of this test, we set the dimension of the box to $[-10,10] R_{E}$ in both $\mathrm{x}$ - and $\mathrm{y}$-directions. For simplicity, we set the parameters constant along the $\mathrm{z}$-direction. The same numerical algorithm from the Raeder global model is used in this simulation. The grid size in this test is $0.1 R_{E}$, which is similar to the grid size that we use later in the global model simulations in the magnetosheath. For the background, we use typical magnetosheath values: $\mathrm{N}=50 \mathrm{~cm}^{-3}, \mathrm{~T}_{\mathrm{p}}=\mathrm{T}_{\mathrm{e}}=100 \mathrm{eV}$, and $\mathrm{B}=50 \mathrm{nT}$ along the $\mathrm{y}$ axis. The initial velocity is set to zero for simplicity. To provide a point wave source, we perturb the plasma pressure at the center of the simulation box with the following form:

$$
\begin{cases}\mathrm{P}=\mathrm{P}_{0}\{0.05[\cos (\pi \mathrm{r})+1.0]+1.0\} & \left(\mathrm{r} \leq 1.0 R_{E}\right), \\ \mathrm{P}=\mathrm{P}_{0} & \left(\mathrm{r}>1.0 R_{E}\right),\end{cases}
$$

where $r=\sqrt{x^{2}+y^{2}}$ is the distance from the center of the box. The initial disturbance of the plasma pressure is shown in Fig. 4 along the $\mathrm{x}$ axis for $\mathrm{y}=0$. The results of this simulation are shown in Fig. 5. In the figure, the fast mode waves and the slow mode waves develop their expected propagation 


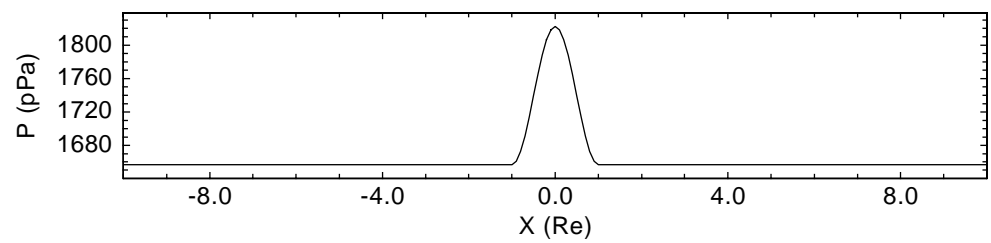

Fig. 4. The initial disturbance of the plasma pressure along the $x$-direction for $y=0$ to test the propagation pattern of the slow mode disturbances and the ability of the global model in describing the slow mode waves in the magnetosheath.
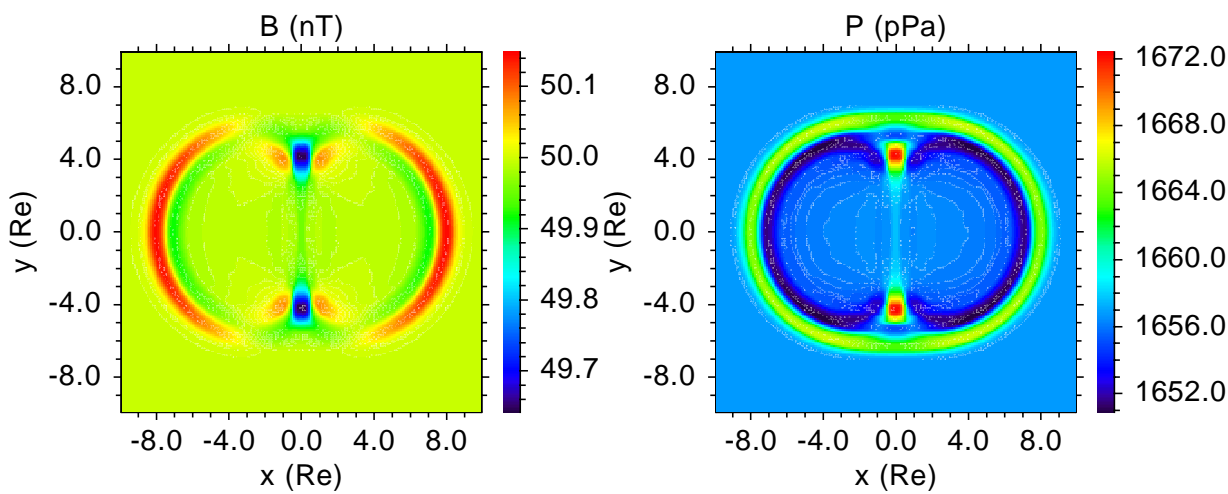

Fig. 5. Results of the slow mode wave test simulation with a small pressure disturbance in the center of the box. The same simulation algorithm and similar grid size are used in this simulation as those in the global simulation in the magnetosheath. Typical magnetosheath values are used to set the uniform background with magnetic field in the y-direction.

patterns from MHD theory. The slow mode waves are shown as the red dots in the pressure panel, and they only propagate along the background magnetic field line with at most a small deviation from that direction. The slow mode wave disturbances from a point source follow the slow mode wave group velocity, instead of the phase velocity, in their propagation and basically reproduce the theoretical slow mode wave Friedrichs II diagram, as shown in Fig. 3. Another important conclusion from this test simulation is that the Raeder global model is sufficient to describe the slow mode formalism in the magnetosheath. Thus, we can use it to address the slow mode waves in this region.

In order to form a slow mode front in the magnetosheath, at each slow mode front location the flow speed must be exactly opposite to the slow mode group velocity in the flow direction. Note here that we do not require significant plasma and/or field changes across a slow mode front, and we describe the plasma and field variations across the front with the effects of it. Figure 6 shows the possible patterns between the flow speed and the slow mode wave group speed, assuming that the slow mode waves can propagate isotropically in all directions. The magnetosheath downstream is on the right-hand side and the upstream is on the left-hand side in each of the two panels. The slow mode wave group speed is shown with dashed lines and the flow speed is shown with solid lines in both panels. Although the slow mode waves can also be generated from the upstream side, to form a standing slow mode front, only the slow mode waves coming from the downstream side need to be considered. In pattern 1 of Fig. 6 , the slow mode wave group speed is smaller than the flow
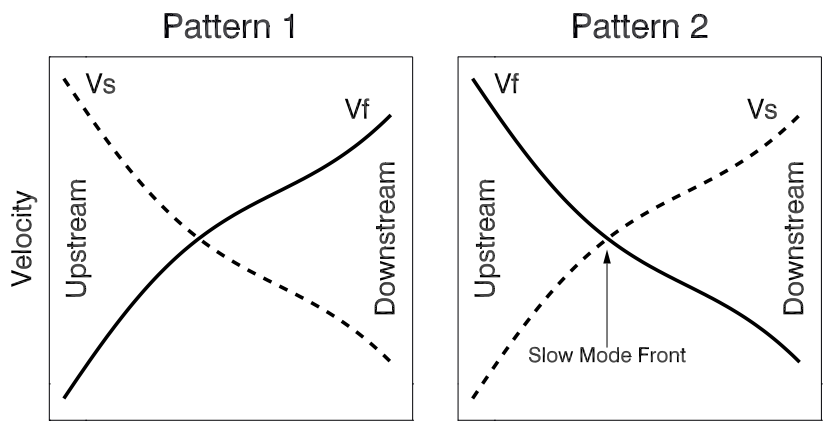

Fig. 6. Two possible patterns between the slow mode wave group speed $\left(\mathrm{V}_{\mathrm{s}}\right)$, assuming no dependence on magnetic field orientation, and the flow speed $\left(\mathrm{V}_{\mathrm{f}}\right)$ in the magnetosheath. The magnetosheath downstream is on the right-hand side and the upstream is on the left-hand side in each of the two panels. The slow mode wave group speed is shown with dashed lines and the flow speed is shown with solid lines. The slow mode front can only exist in pattern 2 .

speed to the right of the crossing point of these two speeds. Thus, as soon as a slow mode wave is generated in the downstream side, it is convected downstream by the flow. In this case no slow mode front can form. Pattern 2 in Fig. 6 is opposite to pattern 1 . The slow mode wave group speed is larger than the flow speed on the downstream side. Thus, the slow mode waves excited in the downstream side can propagate upstream until they reach a stagnation point where the slow mode wave group speed and the flow speed exactly cancel each other. At this point, the slow mode front exists. 

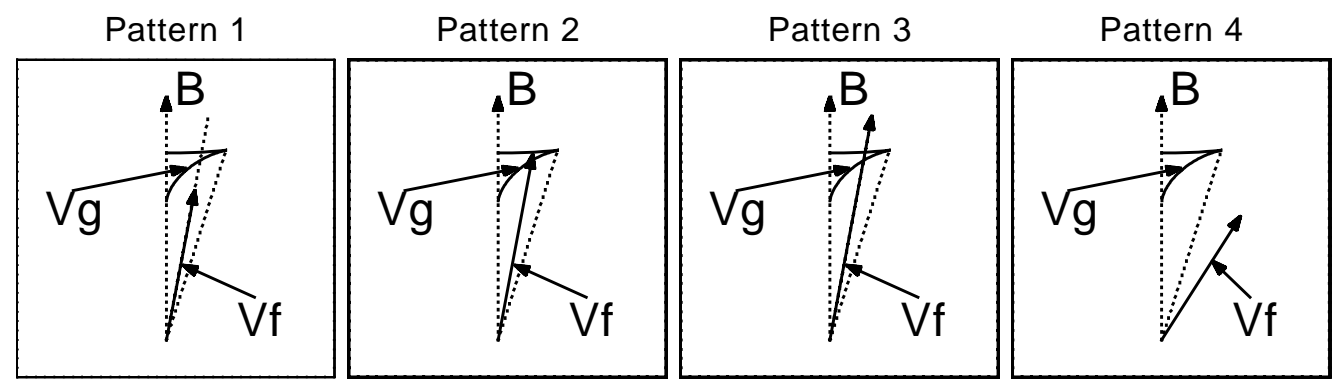

Fig. 7. Four more realistic possible patterns between the slow mode wave group velocity and the flow velocity. The background magnetic field is in the upward direction in all these panels. Only partial slow mode wave group velocity is shown in each panel for simplicity. In pattern 1, the slow mode wave group velocity can overcome the flow velocity, thus the slow mode waves can propagate upstream along the flow line. In patterns 3 and 4, the slow mode wave group velocity cannot overcome the flow velocity, thus the slow mode waves are taken downstream by the flow. Pattern 2 is between pattern 1 and patterns 3 and 4, where the flow velocity and the slow mode wave group velocity cancel each other in the flow direction. The slow mode front occurs where pattern 2 exists.

Table 1. Input solar wind plasma and IMF parameters in the GSE coordinate for the global model runs in this study. Here $\mathrm{M}_{\mathrm{F}}$ is the fast mode Mach number and $\mathrm{M}_{\mathrm{A}}$ is the Alfvén Mach number.

\begin{tabular}{lccccccc}
\hline Run No. & $\boldsymbol{V}(\mathrm{km} / \mathrm{s})$ & $\boldsymbol{B}(\mathrm{nT})$ & $\mathrm{N}\left(\mathrm{cm}^{-3}\right)$ & $\mathrm{T}_{\mathrm{p}}=\mathrm{T}_{\mathrm{e}}(\mathrm{eV})$ & $\mathrm{M}_{\mathrm{F}}$ & $\mathrm{M}_{\mathrm{A}}$ & $\beta$ \\
\hline Run 1 & $(-450,0,0)$ & $(0,0,7)$ & 6 & 10 & 5.3 & 7.2 & 0.99 \\
Run 2 & $(-450,0,0)$ & $(0,0,14)$ & 6 & 10 & 3.3 & 3.6 & 0.25 \\
Run 3 & $(-450,0,0)$ & $(7,0,7)$ & 6 & 10 & 4.3 & 5.1 & 0.49 \\
\hline
\end{tabular}

The real situation is more complex because of the dependence of the slow mode wave group velocity on the background magnetic field orientation. More realistic patterns between the slow mode wave group velocity and the flow velocity are shown in Fig. 7. Note that only a short section of the slow mode group velocity is shown in each panel. For the complete slow mode wave group velocity, please refer Fig. 3. In pattern 1 of Fig. 7, the flow velocity is smaller than the slow mode wave group velocity in the flow direction, thus a slow mode wave can propagate upstream of the flow along the flow line. In pattern 3 of Fig. 7, the flow velocity is larger than the slow mode wave group velocity in the flow direction. In this case, slow mode wave cannot propagate upstream of the flow but is convected downstream. In pattern 4 of Fig. 7 , although the slow mode wave group velocity can be larger in magnitude than the flow velocity, it can not propagate in the flow direction. As a result, the slow mode wave is also convected downstream by the flow. Pattern 2 of Fig. 7 is between pattern 1 and patterns 3 and 4, where the flow velocity and the slow mode wave group velocity cancel each other in the flow direction. The slow mode front occurs where pattern 2 exists. Whether the slow mode front can significantly change the flow and field in the magnetosheath, or whether it can steepen to form a slow mode shock, will be discussed later in this paper.

\section{Model}

The Raeder global geospace model is used in this study. This model solves resistive MHD equations with isotropic pressure in a large three-dimensional volume surrounding the Earth, such that the entire interaction region between the solar wind and the magnetosphere, as well as the ionosphere, is included (Raeder, 2003). Specifically, the simulation domain comprises the bow shock, the magnetopause, and the magnetotail up to several hundred $R_{E}$ from the Earth. The NOAA Coupled Thermosphere Ionosphere Model (CTIM) has been included to handle the coupling between the magnetosphere and the ionosphere (Fuller-Rowell et al., 1996; Raeder et al., 2001). In this study, however, we only use a uniform ionosphere conductivity of 5 Siemens because the ionosphere is likely to have very little influence on the magnetosheath flow.

The Raeder global model can be driven by both observed and idealized solar wind plasma and IMF conditions. In our former PDL case study for model validation, we used spacecraft solar wind observations to drive the model and then compared model results with in-situ PDL observations (Wang et al., 2003). In another study of the magnetosheath structures and forces, we used idealized constant solar wind conditions to expose the basic physical processes of the magnetosheath and the PDL without distracting solar wind oscillations and structures (Wang et al., 2004). In this study, we will continue to use idealized constant solar wind conditions. The input parameters for the base run, Run 1, are shown in Table 1. These parameters are typical solar wind 


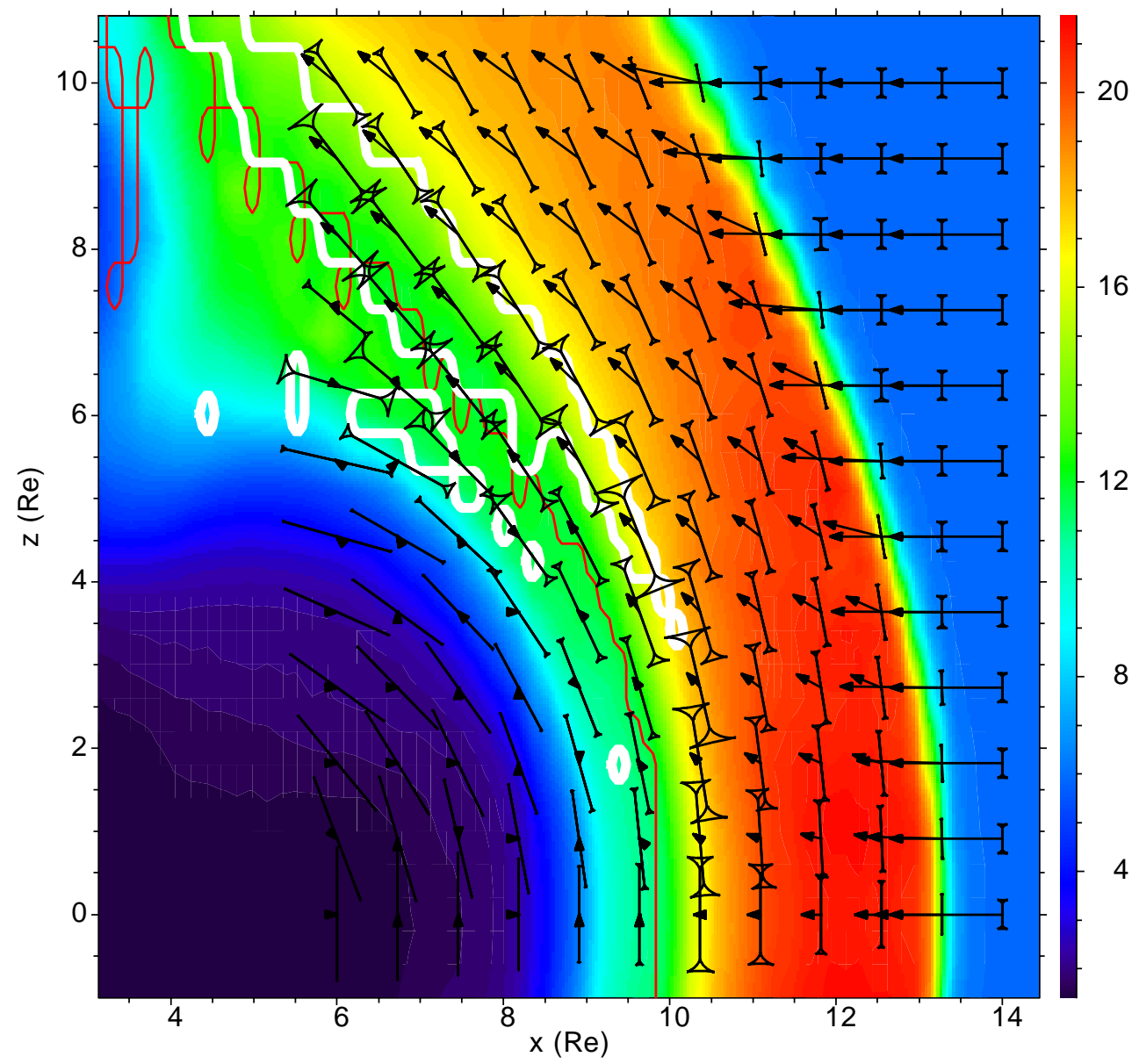

Fig. 8. The slow mode front in the GSE $y=0$ plane with the plasma density as the background for Run 1 . The open-closed magnetic field boundary is shown as a red zigzag curve, which is defined as the boundary between field lines, with both ends connecting to the Earth and field lines with only one end connecting to the Earth. The black arrows show the flow velocity and the black straight lines are along the local magnetic field with the slow mode wave group velocities on the ends of them. The closed white zigzag curves fit pattern 2 of Fig. 7 inside of which pattern 1 in Fig. 7 exists. The segment of a white curve where plasma flows into the region circled by the white curve is a slow mode front.

values except for the northward IMF, and they are the same set of parameters as that used in Wang et al. (2004). In order to show the dependence of the slow mode front on solar wind conditions, we also make two other runs, Run 2 and Run 3, whose solar wind input parameters are also listed in Table 1. A systematic study of the PDL dependence on solar wind conditions and the Earth dipole tilt will be left for another paper. The Raeder global model uses a stretched Cartesian grid. In this study, we have $\sim 22$ grid points between the bow shock and the magnetopause along the Sun-Earth line. This leads to a spatial resolution of $\sim 0.15 R_{E}$ in the subsolar magnetosheath region, which should be sufficient to resolve the PDL with typical observed thickness of $\sim 0.3-1.0 R_{E}$. All the model results shown here are obtained three hours after the start of each global model run when stable magnetosheath structures have already been developed.

\section{Results}

\subsection{The slow mode front in the magnetosheath}

Figure 8 shows the slow mode front in the GSE $y=0$ plane with the plasma density as the background for Run 1 . The open-closed magnetic field boundary is shown as a red zigzag curve, which is defined as the boundary between field lines, with both ends connecting to the Earth and field lines with only one end connecting to the Earth. The complex magnetic field line connectivity near the cusp region is likely caused by the complex flow, and field structures and reconnection. The black arrows show the flow velocity and the black straight line segments are along the local magnetic field with local slow mode wave group velocities on both ends of them. We use local plasma parameters at each grid point to calculate the slow mode group velocity and the technique introduced in Sect. 2 to calculate the slow mode front. The closed white zigzag curves fit pattern 2 of Fig. 7, in which case the flow 

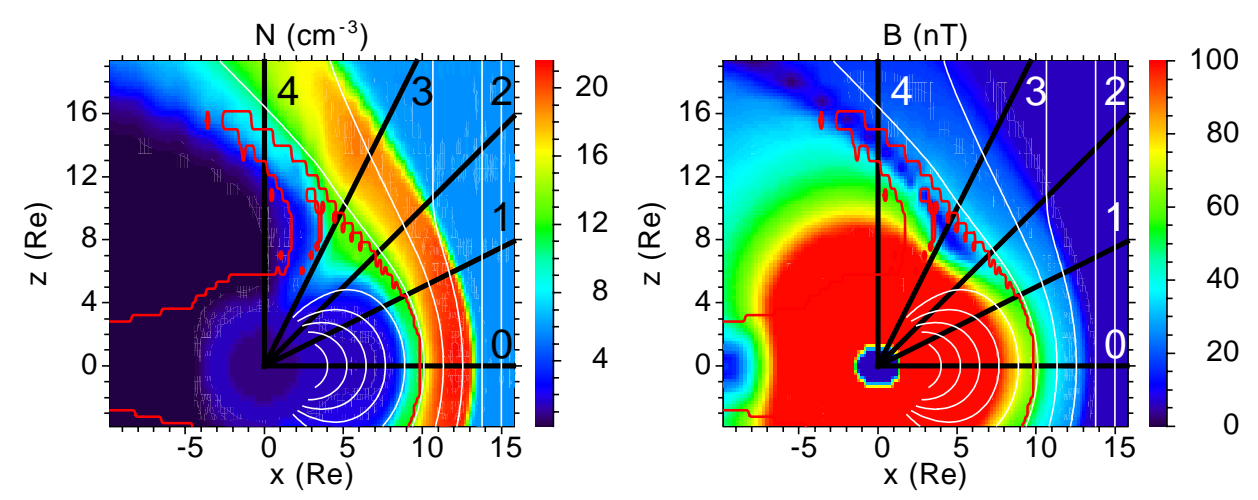

Fig. 9. The plasma density and the magnetic field magnitude in the GSE $y=0$ plane for Run 1 . The open-closed magnetic field boundary is shown as a red zigzag curve and magnetic field lines are shown as white smooth curves in each of the panels. We draw several radially outward straight lines from the center of the Earth. Some plasma and field values along radial lines 0 and 1 are shown in Fig. 10.

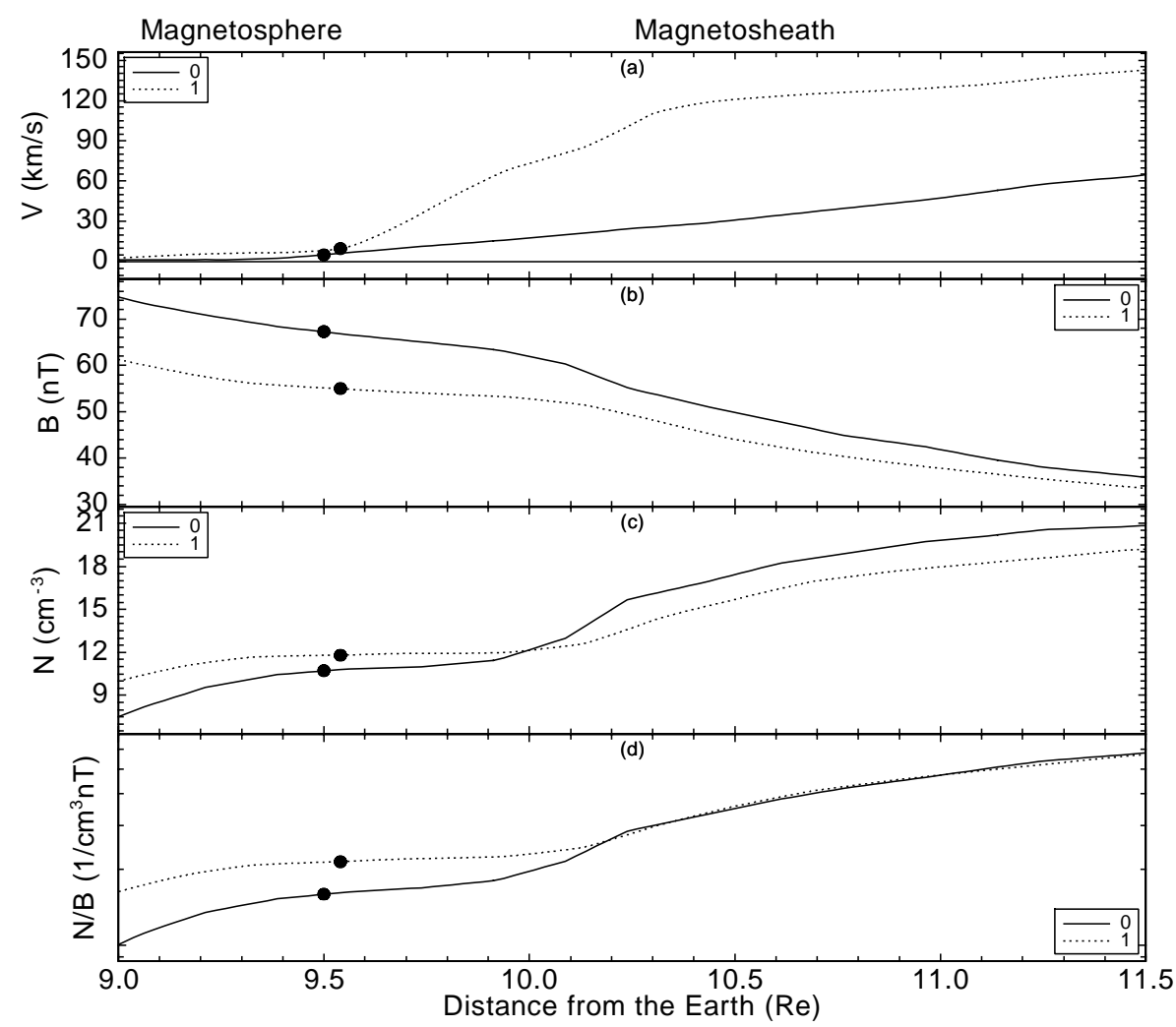

Fig. 10. Some parameters along lines 0 and 1 in Fig. 9. The black dots mark the locations of the magnetopause where the flow speed approaches zero.

velocity and the slow mode wave group velocity cancel each other along the flow direction. Inside the regions circled by the white curves, the slow mode waves can propagate faster than the flow speed in the flow direction, while the opposite occurs outside of these regions. The segments of the white curves where plasma flows into the regions circled by the white curves are the slow mode fronts. In Fig. 8, a clear configuration between the flow velocity and the slow mode wave group velocity can be seen at each location that we choose. A slow mode front exists in the magnetosheath with a distance of $\sim 4 R_{E}$ away from the GSE $\mathrm{z}=0$ plane and $\sim 1 R_{E}$ away from the open-closed magnetic field boundary. This result confirms the prediction of Southwood and Kivelson (1992) for the existence of the slow mode front in the magnetosheath, at least for the solar wind conditions used for Run 1. However, as we will show later, little variations of plasma and field parameters across the front mean that the front has little effect on the flow and field in the magnetosheath. 

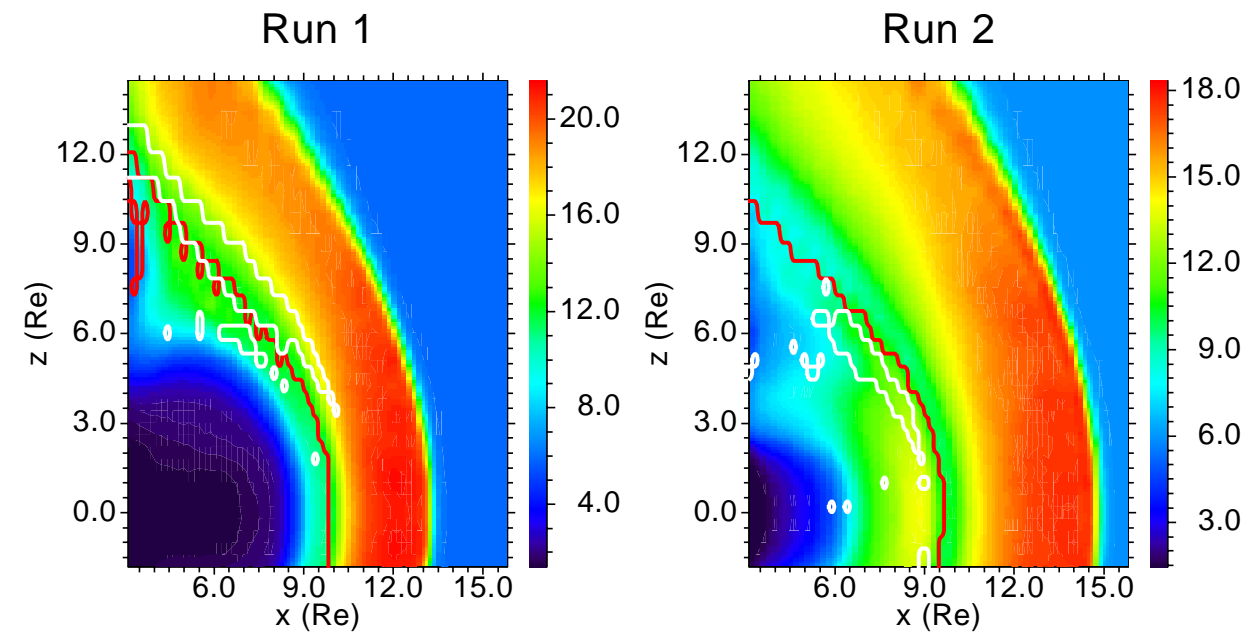

Fig. 11. Comparison between the slow mode fronts in the GSE $y=0$ plane for Run 1 and Run 2. The open-closed magnetic field boundary is shown as a red zigzag curve and the slow mode fronts are shown as closed white zigzag curves (only the segments of the white curves where flow moves into the closed regions should be identified as the slow mode fronts) in each of the panels.

\subsection{The dependence of the PDL on the slow mode front}

Figure 9 shows the plasma density and the magnetic field magnitude in the GSE $y=0$ plane for Run 1 . The open-closed magnetic field boundary is shown as a red zigzag curve and magnetic field lines are shown as white smooth curves in each of the panels. Very clear PDL structure on the magnetopause is seen with decreased plasma density and enhanced magnetic field magnitude. We draw several radially outward straight lines from the center of the Earth in each panel of Fig. 9. Some parameters along radial straight lines 0 and 1 are shown in Fig. 10. On lines 2-4 of Fig. 9, the cusp effects are involved, thus we do not show the parameters on these lines. In Fig. 10, the black dots mark the locations of the magnetopause where the flow speed approaches zero. We see PDL structures on the magnetopause along lines 0 and 1 in this figure with decreased plasma density and enhanced magnetic field magnitude compared to their upstream magnetosheath values. No density enhancement occurs outside of the PDL. For a more detailed discussion about similar figures in some other aspects, please see Wang et al. (2004). Now let us concentrate on the relation between the slow mode front and the PDL, keeping in mind that the results shown in Figs. 8, 9, and 10 are from the same run in the GSE $y=0$ plane. In Fig. 10, there is an obvious PDL structure along line 0, which is the Sun-Earth line. However, no slow mode structure with enhanced plasma density and decreased magnetic field magnitude is seen along this line. This means that the slow mode front, as proposed by Southwood and Kivelson (1992), is not a necessary condition for the formation of the PDL, at least for the stable solar wind conditions used in Run 1. Instead, the PDL is the net result of the combined MHD forces in the magnetosheath (Wang et al., 2004). Meanwhile, only one-layered conventional PDL structure, instead of the two-layered slow mode structure, is seen along this line. The above results hold true for many other tests with different solar wind conditions and different model resolutions, which we do not show here.

\subsection{The dependence of the slow mode front on solar wind conditions}

After confirming the existence of the slow mode front in the magnetosheath, an interesting question is: what is the dependence of such a structure on solar wind conditions? Figure 11 shows the comparison between the slow mode fronts in the GSE $y=0$ plane for Run 1 and Run 2. Solar wind input parameters for these two model runs are listed in Table 1 and the only difference between them is the IMF $B_{z}: 7 \mathrm{nT}$ for Run 1 and $14 \mathrm{nT}$ for Run 2. For Run 1, slow mode fronts exist sunward of the open-closed magnetic field boundary in the magnetosheath. However, for Run 2, slow mode fronts do not exist outside of the open-closed magnetic field boundary. Meanwhile, the slow mode fronts are more shrunken in Run 2 than in Run 1. Some parameters along the Sun-Earth line for Run 2 are shown in Fig. 12, from which we can also see clear PDL structure on the magnetopause with decreased plasma density and enhanced magnetic field comparing to their upstream values. This further confirms that the slow mode front does not play an important role for the formation of the PDL.

Figure 13 shows the slow mode front in the magnetosheath in the GSE $\mathrm{y}=0$ plane for Run 3, a case with a finite IMF $B_{x}$. The plasma density is shown as the background, the open-closed magnetic field boundary is shown as a red zigzag curve, magnetic field lines are shown as white smooth curves, and the slow mode fronts are shown as closed black zigzag curves (only the segments of the curves where flow moves into the closed regions should be identified as the slow mode fronts). The field lines pile up on the magnetopause in a 


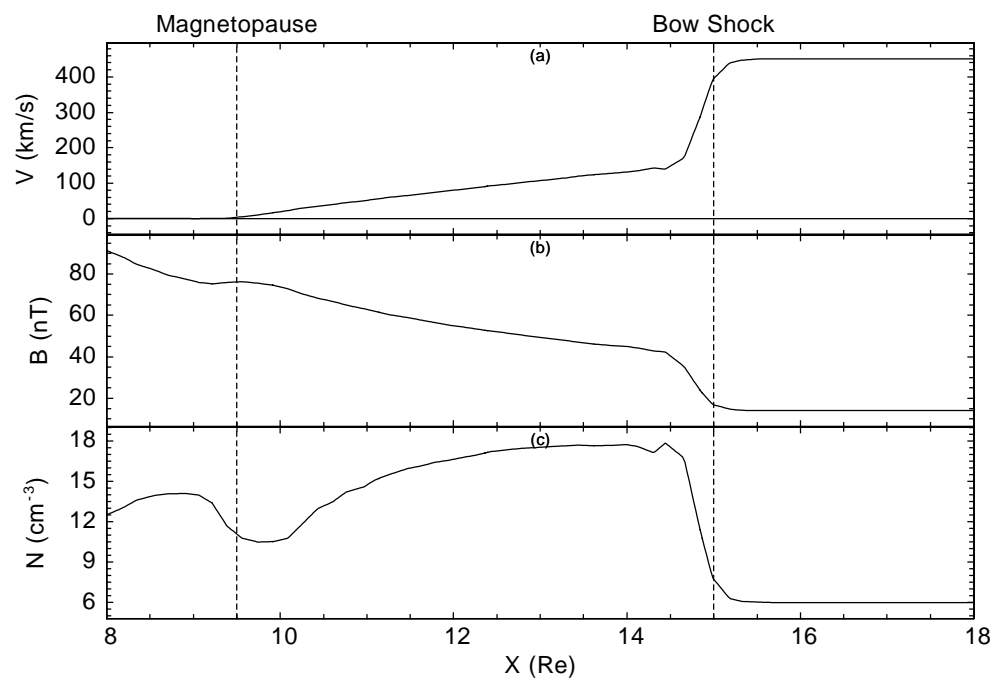

Fig. 12. Some parameters along the Sun-Earth line for Run 2. From top to bottom are: the flow speed, the magnetic field magnitude, and the plasma density. The magnetopause is defined as the location where the flow speed in the magnetosheath approaches zero.

different way than Run 1 because the field symmetry in the magnetosheath is broken. As a result, the structures of the slow mode front in the magnetosheath are drastically different from those of Run 1 and Run 2. Specifically, there is a large slow mode front below the GSE $\mathrm{z}=0$ plane which is as close as $\sim 2 R_{E}$ away from it. Meanwhile, the slow mode fronts above the GSE $\mathrm{z}=0$ plane shrink to a very small one, which is $\sim 6 R_{E}$ away from the GSE z=0 plane. We draw a green flow line from the solar wind crossing the magnetosheath slow mode front below the GSE $\mathrm{z}=0$ plane. Some parameters along this green flow line are shown in Fig. 14. The solar wind is on the left-hand side and the magnetosheath is on the right-hand side of Fig. 14. The horizontal axis is the distance along the flow line from its starting point at $(15,0$, $-2.5) R_{E}$, and the big jump at $\sim 2 R_{E}$ is the bow shock. The black dot on each line corresponds to the black dot in Fig. 13 which marks the location where the green flow line crosses the slow mode front. An important criteria for a slow mode shock is the dissipation across the slow mode front. If a clear energy dissipation is seen across the front (which can be easily seen from temperature profile), we can say that the slow mode front has developed into a slow mode shock. In Fig. 14, at the point where the flow line crosses the slow mode front, the parameters are smooth with no indication of energy dissipation. A blowup figure around the black dot in Fig. 14 is shown in Fig. 15, still with no signature of noticeable energy dissipation. This result confirms that no slow mode shock is developed at the slow mode front. Further, the flow and field patterns in the magnetosheath are not affected by the slow mode front in a noticeable way.

\section{Discussion and conclusions}

We propose a new numerical method to find the location of the slow mode front in the magnetosheath by analyzing the relative configuration between the slow mode wave group velocity and the flow velocity. We find the existence of the slow mode front in the magnetosheath in normal solar wind conditions with northward IMF, which is in line with the prediction by Southwood and Kivelson (1992). In this slow mode front detection technique, we assume a uniform background for the slow mode group velocity calculation, which does not fit exactly in the magnetosheath because of the plasma and field gradients. However, the results of some extensive simulations, similar to what we have done for Fig. 5 but with typical magnetosheath gradients, have shown no significant differences.

Figure 2 shows a sketch of a rationalized scenario for the flow structure upstream of the subsolar magnetopause. There is a field compression region with roughly the properties of Zwan and Wolf's flux tube immediately adjacent to the magnetopause. However, the field is not aligned with the outer boundary of the region. Field lines threading the compression region bend toward the Sun and enter a field rarefaction region which is immediately behind a slow MHD wave shock/front (Southwood and Kivelson, 1992). Outside the front the field threads the incoming magnetosheath flow. By comparing Fig. 2 with Fig. 13, we see that the slow mode front structures from our global model results are much more complex than the schematic slow mode front structures in the theoretical model. Further, the fronts occur at different locations. In Fig. 2, the slow mode front is drawn where magnetic field lines tilt toward the Sun. However, in our model results, the slow mode front appears where magnetic field lines tilt away from the Sun. In the latter configuration, the slow mode velocity is more aligned with the flow velocity, and thus it is easier for the slow mode wave to propagate upstream to form a slow mode front.

In our model results the slow mode front does not exist in the magnetosheath for all solar wind conditions. In some regions where the PDL exists there is no slow mode front that 


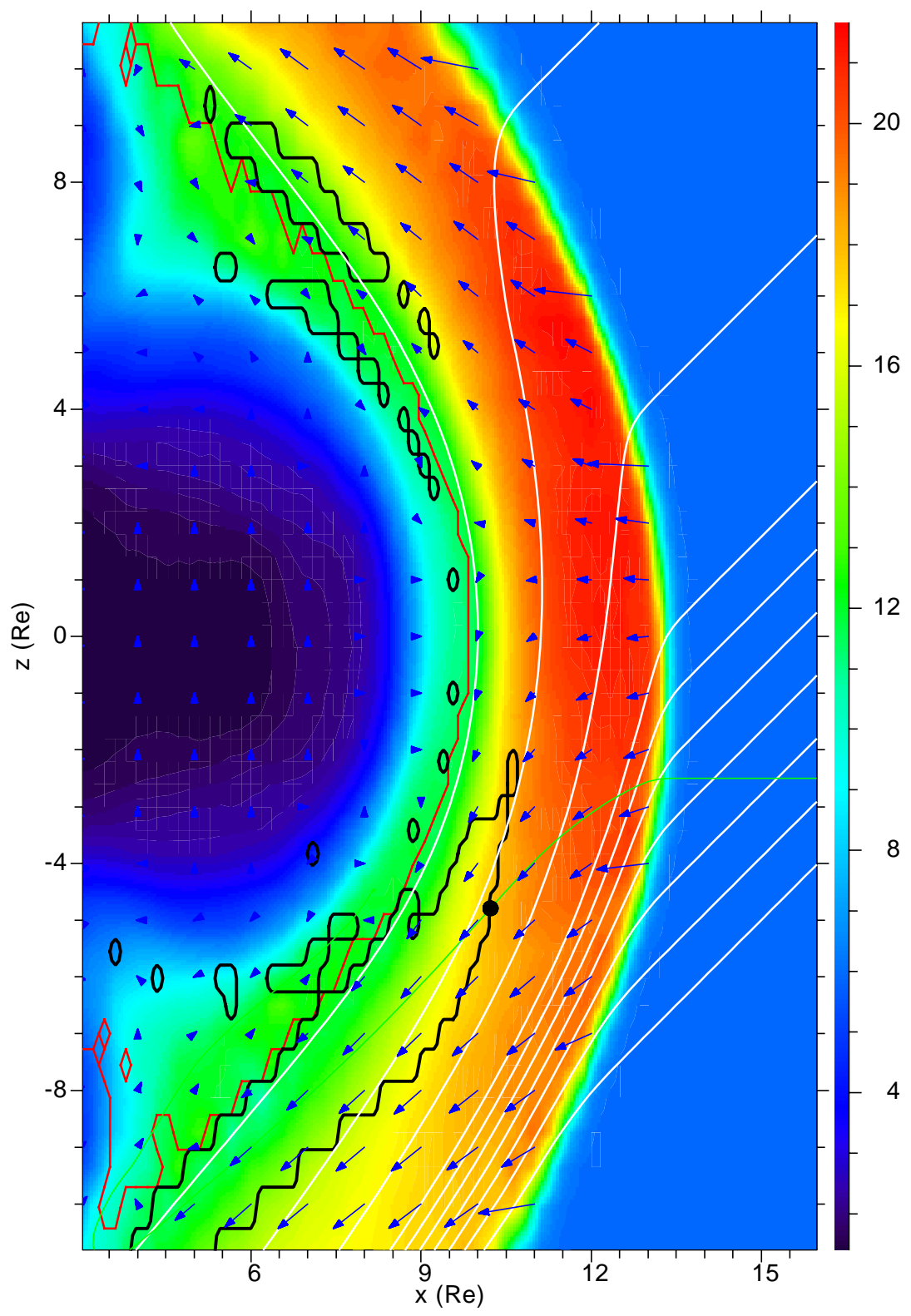

Fig. 13. The slow mode fronts in the magnetosheath in the GSE $y=0$ plane for Run 3 with tilted IMF. The plasma density is shown as the background, the open-closed magnetic field boundary is shown as a red zigzag curve, magnetic field lines are shown as white smooth curves, and the blue arrows are the flow velocity. The slow mode fronts are shown as closed black zigzag curves (only the segments of the curves where plasma flows into the closed regions should be identified as the slow mode fronts). Some parameters along the green flow line which crosses the slow mode front are shown in Fig. 14.

could cause it. Instead of steepening and developing into a slow mode shock or causing big flow and field variations, the slow mode front has no discernible influence on the magnetosheath plasma and field patterns for the solar wind conditions used in our several model runs. These results imply that the slow mode front, at most, plays an insignificant role for the PDL formation. We believe the combined pressure gradient force and magnetic force play the critical role in shaping the magnetosheath environment (Wang et al., 2004).

The differences between our results and the results by Southwood and Kivelson (1995) reflect the different assump- tions in these two models. Although Southwood and Kivelson used MHD theory and the properties of the slow mode wave to explain the formation of the PDL, they had to make assumptions about the magnetosheath flow pattern and magnetic field topology, as well as a slow mode wave source near the subsolar magnetopause. As we have demonstrated here, the full-scale, nonlinear solution of the MHD equations is required to obtain a realistic picture of the field, flow, and governing forces in the magnetosheath.

Our results also show that two-layered slow mode structures do not exist in the magnetosheath along the radial lines 


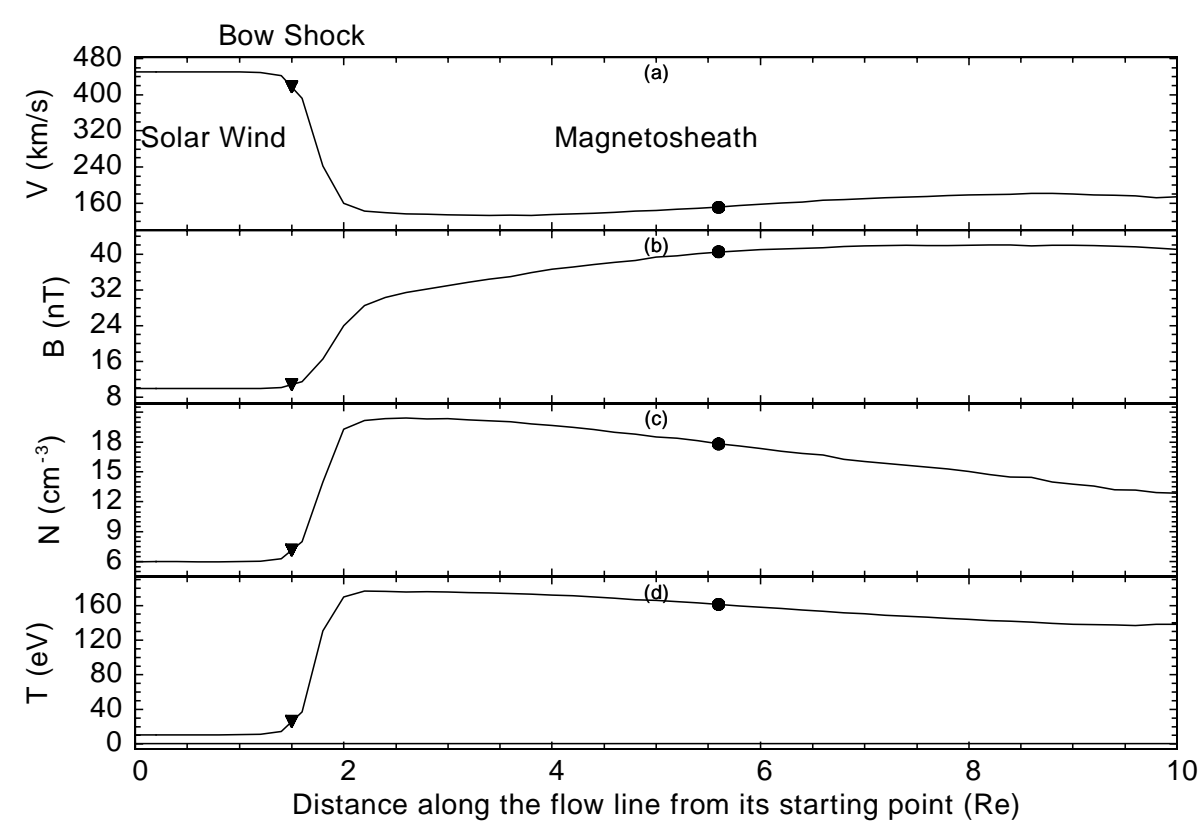

Fig. 14. Some parameters along the green flow line in Fig. 13. The solar wind is on the left-hand side and the magnetosheath is on the right-hand side. The horizontal axis is the distance along the flow line from its starting point at $(15,0,-2.5) R_{E}$. The big jump at $\sim 2 R_{E}$ is the bow shock. The black dot on each line corresponds to the black dot in Fig. 13 which marks the location where the flow line crosses the slow mode front.

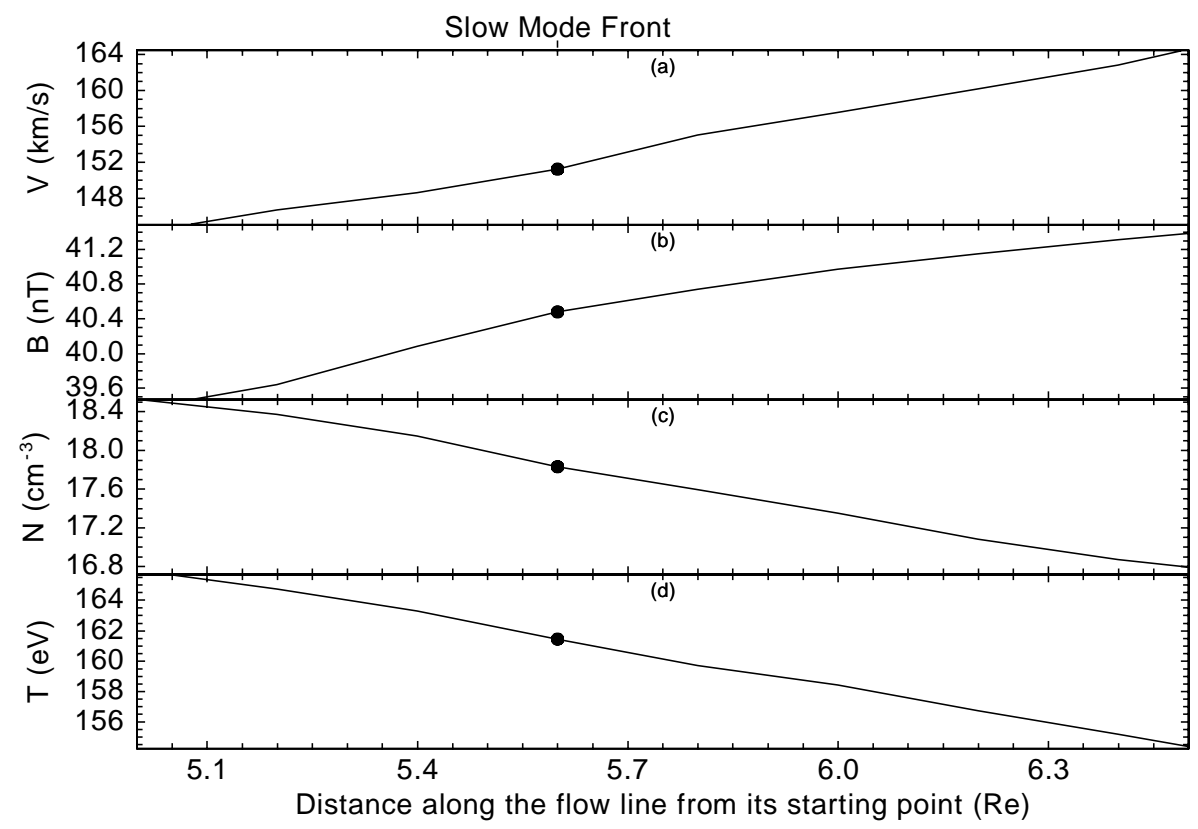

Fig. 15. A blowup of Fig. 14 around the black dot in the magnetosheath for a better view of the plasma and field transitions across the slow mode front along the green flow line in Fig. 13.

from the Earth for the solar wind conditions used in this study. However, it is too early to say that the two-layered slow mode structures do not exist in some other cases. In the complex and dynamically varying magnetosheath geometry and force environment, it is still possible for the formation of a two-layered slow mode structure. One scenario is that plasma flow coming from one magnetosheath region causes expansion in another magnetosheath region, thus pushing away magnetic field lines. After this, a region with decreased magnetic field and increased plasma density should be found, which could be the non-PDL slow mode structure in the observed two-layered slow mode structure on the magnetopause. Another factor that can cause complex magnetosheath structures is the dynamically changing solar wind conditions. Magnetosheath structures depend strongly on solar wind conditions (e.g. Wang et al., 2003). Many 
observed structures in the magnetosheath reflect solar wind variations. Thus, complex structures, like two-layered slow mode structures, could also be seen as a result. This is the reason why concurrent solar wind observations are necessary to tell whether a structure in the magnetosheath is of solar wind origin. Finally, among the 26 ISEE 1 and 2 magnetopause passes near the stagnation line in Song et al. (1990), 17 events show slow mode signatures with plasma density enhancements. However, only 4 of these 17 events correspond to northward IMF, while the others occurred during southward IMF or the IMF $B_{z}$ close to zero when more disturbance in the magnetosheath is expected. The more disturbed magnetosheath environment is likely to produce more slow mode waves, and thus enhances the role of the slow mode waves for controlling the flow and field patterns in the magnetosheath. A detailed study in this direction will help us to clarify this assumption, which is beyond the scope of this paper.

The primary purpose of this paper has been to determine whether the slow mode front can exist in the magnetosheath, and if so, what possible role it plays for the formation of the PDL. Using global simulations we reach the following conclusions:

1. We have introduced a new numerical method to calculate the slow mode front in the magnetosheath that compares the relative pattern between the slow mode wave group velocity and the flow velocity. The model results show that the slow mode front exists in the magnetosheath for certain, but not all, solar wind conditions, which is consistent with the prediction of Southwood and Kivelson $(1992,1995)$.

2. The slow mode front in our simulations has more complex structures than that from the Southwood and Kivelson (1995) model. In particular, a very different slow mode front geometry is found in our model results, compared to the Southwood and Kivelson (1995) model picture. The front shows a clear dependence on the IMF $B_{z}$ and $B_{x}$, but it does not develop into a shock and has little effect on the flow and field in the magnetosheath.

3. For the stable solar wind conditions that we used, the PDL structure does not necessarily correspond to the slow mode front as proposed by Southwood and Kivelson (1992), and we do not see the two-layered slow mode structures as observed by Song et al. $(1990,1992)$. We believe that the combined MHD forces determine the formation of the PDL.

Acknowledgements. The authors of the paper would like to thank M. G. Kivelson and R. J. Strangeway for their helpful discussions, as well as the two reviewers for their helpful comments to improve this paper. This work was supported by grant ATM-00-84483 and grant ATM-01-01145 from the National Science Foundation and grant NAG 5-10986 from the National Aeronautics and Space Administration. Computations were performed at San Diego Supercomputer Center. IGPP publication number 5798.
Topical Editor T. Pulkkinen thanks M. Witberger and another referee for their help in evaluating this paper.

\section{References}

Anderson, B. J. and Fuselier, S. A.: Magnetic pulsations from 0.1 to $4.0 \mathrm{~Hz}$ and associated plasma properties in the Earth's subsolar magnetosheath and plasma depletion layer, J. Geophys. Res., 98, 1461-1479, 1993.

Anderson, B. J., Fuselier, S. A., Gary, S. P., and Denton, R. E. Magnetic spectral signatures in the Earth's magnetosheath and plasma depletion layer, J. Geophys. Res., 99, 5877-5891, 1994.

Anderson, B. J., Phan, T. D., and Fuselier, S. A.: Relationships between plasma depletion and subsolar reconnection, J. Geophys. Res., 102, 9531-9542, 1997.

Crooker, N. U., Eastman, T. E., and Stiles, G. S.: Observations of plasma depletion in the magnetosheath at the dayside magnetopause, J. Geophys. Res., 84, 869-874, 1979.

Cummings, W. D. and Coleman, P. J.: Magnetic fields in the magnetopause and vicinity at synchronous orbit, J. Geophys. Res., 73, 5699-5718, 1968.

Denton, R. E. and Lyon, J. G.: Effect of pressure anisotropy on the structure of a two-dimensional magnetosheath, J. Geophys. Res., 105, 7545-7556, 2000.

Fuller-Rowell, T. J., Rees, D., Quegan, S., Moffett, R. J., Codrescu, M. V., and Millward, G. H.: A coupled thermosphere-ionosphere model (CTIM), in: STEP Report, edited by Schunk, R., Scientific Committee on Solar Terrestrial Physics (SCOSTEP), NOAA/NGDC, Boulder, Colorado, 217-238, 1996.

Fuselier, S. A., Klumpar, D. M., Shelley, E. G., Anderson, B. J., and Coates, A. J.: $\mathrm{He}^{2+}$ and $\mathrm{H}^{+}$dynamics in the subsolar magnetosheath and plasma depletion layer, J. Geophys. Res., 96, 21 095-21 104, 1991.

Lyon, J. G.: MHD simulations of the magnetosheath, Adv. Space Res., 14, 21-28, 1994.

Paschmann, G., Baumjohann, W., Sckopke, N., Phan, T. D., and Lühr, H.: Structure of the dayside magnetopause for low magnetic shear, J. Geophys. Res., 98, 13 409-13 422, 1993.

Phan, T. D., Paschmann, G., Baumjohann, W., Sckopke, N., and Lühr, H.: The magnetosheath region adjacent to the dayside magnetopause: AMPTE/IRM observations, J. Geophys. Res., 99, 121-141, 1994.

Phan, T. D., Larson, D., McFadden, J., Carlson, C., Moyer, M., Paularena, K. I., McCarthy, M., Parks, G. K., Réme, H., Sanderson, T. R., and Lepping, R. F.: Low-latitude dusk flank magnetosheath, magnetopause, and boundary layer for low magnetic shear: Wind observations, J. Geophys. Res., 102, 19883-19895, 1997.

Raeder, J.: Global magnetohydrodynamics - A tutorial, in: Space Plasma Simulation, edited by Buchner, J., Dum, C. T., and Scholer, M., Springer Verlag, Heidelberg, Lecture Notes in Physics, Volume 615, 212-246, 2003.

Raeder, J., Wang, Y. L., and Fuller-Rowell, T. J.: Geomagnetic storm simulation with a coupled magnetosphere-ionospherethermosphere model, in: Space Weather, edited by Song, P., Singer, H. J., and Siscoe, G. L., Geophysical Monograph Series, Volume 125, 377-384, 2001.

Siscoe, G. L., Crooker, N. U., Erickson, G. M., Sonnerup, B. U. Ö., Maynard, N. C., Schoendorf, J. A., Siebert, K. D., Weimer, D. R., White, W. W., and Wilson, G. R.: MHD properties of magnetosheath flow, Planet. Space Sci., 50, 461-471, 2002. 
Song, P., Russell, C. T., Gosling, J. T., Thomsen, M., and Elphic, R. C.: Observations of the density profile in the magnetosheath near the stagnation streamline, Geophys. Res. Lett., 17, 20352038, 1990.

Song, P., Russell, C. T., and Thomsen, M. F.: Slow mode transition in the frontside magnetosheath, J. Geophys. Res., 97, 82958305, 1992.

Song, P., Russell, C. T., Fitzenreiter, R. J., Gosling, J. T., Thomsen, M. F., Mitchell, D. G., Fuselier, S. A., Parks, G. K., Anderson, R. R., and Hubert, D.: Structure and properties of the subsolar magnetopause for northward interplanetary magnetic field: Multiple-instrument particle observations, J. Geophys. Res., 98, $11319-11337,1993$.

Southwood, D. J. and Kivelson, M. G.: On the form of the flow in the magnetosheath, J. Geophys. Res., 97, 2873-2879, 1992.
Southwood, D. J. and Kivelson, M. G.: Magnetosheath flow near the subsolar magnetopause: Zwan-Wolf and SouthwoodKivelson theories reconciled, Geophys. Res. Lett., 22, 32753278, 1995.

Wang, Y. L., Raeder, J., Russell, C. T., Phan, T. D., and Manapat, M.: Plasma depletion layer: Event studies with a global model, J. Geophys. Res., 108, 1010, doi:10.1029/2002JA009281, 2003.

Wang, Y. L., Raeder, J., and Russell, C. T., Plasma depletion layer: Magnetosheath flow structure and forces, Annales Geophysicae, 22, 1001-1017, 2004.

Wu, C. C.: MHD flow past an obstacle: Large-scale flow in the magnetosheath, Geophys. Res. Lett., 19, 87-90, 1992.

Zwan, B. J. and Wolf, R. A.: Depletion of solar wind plasma near a planetary boundary, J. Geophys. Res., 81, 1636-1648, 1976. 